\title{
Effects of ion solvation on phase equilibrium and interfacial tension of liquid mixtures
}

\author{
Rui Wang and Zhen-Gang Wanga) \\ Division of Chemistry and Chemical Engineering, California Institute of Technology, Pasadena, \\ California 91125, USA
}

(Received 30 April 2011; accepted 10 June 2011; published online 7 July 2011)

\begin{abstract}
We study the bulk thermodynamics and interfacial properties of electrolyte solution mixtures by accounting for electrostatic interaction, ion solvation, and inhomogeneity in the dielectric medium in the mean-field framework. Difference in the solvation energy between the cations and anions is shown to give rise to local charge separation near the interface, and a finite Galvani potential between two coexisting solutions. The ion solvation affects the phase equilibrium of the solvent mixture, depending on the dielectric constants of the solvents, reflecting the competition between the solvation energy and translation entropy of the ions. Miscibility is decreased if both solvents have low dielectric constants and is enhanced if both solvents have high dielectric constant. At the mean-field level, the ion distribution near the interface is determined by two competing effects: accumulation in the electrostatic double layer and depletion in a diffuse interface. The interfacial tension shows a nonmonotonic dependence on the salt concentration: it increases linearly with the salt concentration at higher concentrations and decreases approximately as the square root of the salt concentration for dilute solutions, reaching a minimum near $1 \mathrm{mM}$. We also find that, for a fixed cation type, the interfacial tension decreases as the size of anion increases. These results offer qualitative explanations within one unified framework for the long-known concentration and ion size effects on the interfacial tension of electrolyte solutions. (C) 2011 American Institute of Physics. [doi:10.1063/1.3607969]
\end{abstract}

\section{INTRODUCTION}

For soft matter and biological systems, ions and electrostatic interactions play an important role in the structural, dynamic, and functional properties. ${ }^{1-4}$ The most common approach to treating electrostatic interactions in the presence of salt ions is the Poisson-Boltzmann (PB) equation. ${ }^{2}$ It is a mean-field theory relating the average electrostatic potential to fixed charge densities and the average concentration of salt ions. Many modifications have been made in the recent years to the PB theory to include effects, such as the excluded volume of the ions, ${ }^{5-7}$ nonlocality of dielectric constants, ${ }^{8-10}$ and the correlation between ions (manifested in the formation of bound ion pairs). ${ }^{11}$

An obvious effect that is missing in the PB theory is the solvation free energy of salt ions in the dissolving medium. While the solvation free energy can be absorbed into a redefinition of a reference state free energy for uniform systems and, therefore, becomes inconsequential, it must play a crucial role in spatially varying dielectric media. In a recent study, one of us developed a new theory that includes the solvation free energy in a general theory for treating fluctuation effects. ${ }^{12}$ The solvation energy is shown to consist of a nonuniversal part in the form of local Born energy and a universal part that depends on the ion concentration, valency, and the dielectric constant. Retaining the local Born energy while ignoring long wavelength fluctuations resulted in an improved mean-field theory, which was termed the Born-energy augmented Poisson-Boltzmann (BEAPB) equation. If anions and

a)Electronic mail: zgw@caltech.edu. cations have different ion radii and/or valency, the BEAPB theory predicts a spatially varying electrostatic potential and local charge separation.

In this work, we study the consequence of the solvation free energy on the miscibility and interfacial tension between two solvents using the BEAPB approach. Experimentally, the dependence of the liquid-gas surface tension on the electrolyte concentration has been measured for a long time. ${ }^{13,14}$ At high salt concentrations, the surface tension exhibits a linear increase with the salt concentration. However, Jones and Ray ${ }^{13}$ showed that in very dilute solutions, the surface tension actually decreases first with the salt concentration and reaching a minimum near $1 \mathrm{mM}$ for several different salt solutions. To date, no theory has satisfactorily explained the full concentration dependence of the surface tension. In addition, the surface tension depends not only on the salt concentration, but also on the identity of cations and anions, which is often interpreted as the specific ion effect. ${ }^{15,16}$ For a fixed cation type, the water-air surface tension decreases according to the Hofmeister sequence: $\mathrm{F}^{-}>\mathrm{Cl}^{-}>\mathrm{Br}^{-}>\mathrm{I}^{-}$. Although there are fewer reported measurements of the liquid-liquid interfacial tension, ${ }^{17}$ its behavior is qualitatively similar to the liquid-gas surface tension, especially concerning the JonesRay effect and the specific ion effect.

Theoretically, great efforts have been made to understand the surface and interfacial tension of electrolyte solutions since the pioneering work of Wagner, ${ }^{18}$ and Onsager and Samaras. ${ }^{19}$ By assuming the absence of ions in the gas phase and considering the image charge repulsion as the dominant effect, Onsager and Samaras obtained the limiting law for the excess liquid-gas surface tension in the form $\Delta \gamma \sim c \ln c$ ( $c$ is 
the salt concentration in bulk solution). However, this theory fails to explain both the Jones-Ray effect and the specific ion effect. Nichols and Pratt ${ }^{20}$ took the presence of ion in the gas phase into account (in this sense, there is no conceptual difference between the liquid-gas surfaces and the liquid-liquid interfaces) and found the excess interfacial tension decreases with the square root of the salt concentration $\Delta \gamma \sim-\sqrt{c}$ for dilute electrolyte solution. Recently, Bier et al. ${ }^{21}$ assumed a shifted steplike form of the solvation potential and obtained analytical results by solving the linearized PB equation. Different scaling behaviors of the interfacial tension observed in experiments can be explained by adjusting the position of the interface in their model. However, the interfacial structure chosen in their work is artificial and the work does not explain the Jones-Ray effect. By means of Ginzburg-Landau approach, Onuki ${ }^{22,23}$ studied the ion distribution and the interfacial tension in the electrolyte mixture near the critical point. Both the solvation energy and the image interaction are taken into account. The behaviors of linear increase and square root decrease of $\Delta \gamma$ with the salt concentration are obtained in his work. However, the solvation energy and the image interaction were introduced phenomenologically, which makes it difficult to connect his model to the behavior of specific ions.

In this work, we provide a unified theoretical framework to describe the effect of ion solvation on the bulk thermodynamics and interfacial properties of electrolyte solution mixture at the mean-field level. We present our general model in Sec. II A by accounting for the electrostatic interactions, the solvation effect and the inhomogeneous dielectric constant. The solvation energy retains the form of Born energy at the mean-field level, which is position-dependent in the inhomogeneous medium. In Sec. II B, we present analytical results for a special case where the composition profile is a sharp steplike function. Some general phenomena caused by the difference in the solvation energy between the cations and anions, such as the finite electrostatic potential difference, the charge separation, and the electrostatic double layer, are also illustrated here. In Sec. III, we examine the effect of adding salt on the miscibility of the solvent mixture. Phase diagrams for different dielectric constants and temperatures are shown. In Sec. IV, we systematically investigate the interfacial structure and the interfacial tension of the electrolyte solution mixture. The effect of electrostatic double layer and the diffuse interface on the ion distribution is examined for different salt concentrations. The dependence of the interfacial tension on salt concentration and ion radius is also studied. Both the Jones-Ray effect and the specific ion effect are captured by our model. Finally, we summarize our key results and discuss possible extensions of the current model in Sec. V.

\section{MODEL AND THEORY}

We consider a binary mixture consisting of solvents $\mathrm{A}$ and $\mathrm{B}$, with dielectric constant $\varepsilon_{\mathrm{A}}$ and $\varepsilon_{\mathrm{B}}$, respectively. We take $\mathrm{A}$ to be the component with higher dielectric constant, and write its volume fraction as $\phi(r)$ to denote the local composition of the mixture. We will use the subscripts $\alpha$ and $\beta$ to denote the A-rich and B-rich phases, respectively, throughout this paper. The salt is composed of simple ions: cations and anions, with $z_{+}$and $z_{+}$denoting their valency (absolute value). In this work, ions are assumed to be volumeless particles; however, they are assigned sizes that capture the charge distribution in the ions. The number concentration of cations and anions are denoted by $c_{+}(r)$ and $c_{-}(r)$, respectively. Here, $\rho(r)=e z_{+} c_{+}(r)-e z_{+} c_{+}(r)$ gives the local charge density, where $e$ is the elementary unit of charge. In the following subsection, we construct the free energy as a functional of the composition, ion concentration, and electrostatic potential in the mean-field framework.

\section{A. Mean-field theory}

We treat our electrolyte solution as an open system: the ions and solvents are in contact with a reservoir of given chemical potentials. The grand potential $G$ of the system can be divided into the electrostatic part $\left(G_{e}\right)$ and the solution part $\left(G_{s}\right)$ as

$$
G=\int d \mathbf{r} g(\mathbf{r})=G_{e}+G_{s}
$$

with $g(\mathbf{r})$ denoting the grand potential density. For the electrostatic part, we consider the Coulomb interaction between different ion species, the interaction between ions and the solvents (i.e., the solvation energy), and the translational entropy of the ions. Other effects, such as the polarization of ions ${ }^{15,24}$ and formation of bound ion pairs ${ }^{11}$ are not taken into account in the present work. Thus, the electrostatic part of the grand potential functional can be written as

$$
\begin{aligned}
G_{e} \equiv & \int d \mathbf{r} g_{e}(\mathbf{r}) \\
= & \int d \mathbf{r}\left[\rho \psi-\frac{\varepsilon_{0} \varepsilon(\phi)}{2}(\nabla \psi)^{2}+c_{+} u_{+}+c_{-} u_{-}\right] \\
& +k T \int d \mathbf{r}\left[c_{+} \ln \left(c_{+} / c_{+}^{R}\right)-c_{+}+c_{-} \ln \left(c_{-} / c_{-}^{R}\right)-c_{-}\right] \\
& -\int d \mathbf{r}\left(c_{+} \mu_{+}+c_{-} \mu_{-}\right),
\end{aligned}
$$

where $\psi$ is the electrostatic potential, $\varepsilon_{0}$ is the vacuum permittivity, $\varepsilon(\phi)$ is the local dielectric constant, $\mu_{+}$and $\mu_{+}$are, respectively, the chemical potential of the cations and anions in the reservoir, and $c_{+}^{R}$ and $c_{-}^{R}$ are their respective reference concentration. Here, $u_{+}$and $u_{-}$are, respectively, the solvation energy of the cations and anions, the form of which will be specified later.

For the solution part of the grand potential, we combine a regular solution expression for homogeneous mixing and a square-gradient expression for the penalty of composition change to write 25,26

$$
\begin{aligned}
G_{s} \equiv & \int d \mathbf{r} g_{s}(\mathbf{r}) \\
= & k T \int d \mathbf{r} \frac{1}{v_{s}}[\phi \ln \phi+(1-\phi) \ln (1-\phi)+\chi \phi(1-\phi)] \\
& +k T \int d \mathbf{r}\left[\frac{1}{2} C(\phi)(\nabla \phi)^{2}-\beta \mu_{s} \phi\right],
\end{aligned}
$$


where $v_{s}$ is the volume of the solvents molecules (assumed for simplicity to be the same for the two solvents) and $\chi$ is the interaction parameter dependent on temperature. $C(\phi)$ scales the composition gradient and is set to be $a_{s}^{2} /\left[18 v_{s} \phi(1-\phi)\right]$ in analogy to binary polymer blends with $a_{s}$ the radius of the solvent molecules ${ }^{27}$ it determines the width of the interface. $\mu_{s}$ is the exchange chemical potential on a per unit volume basis (i.e., $\left.\mu_{s}=\left(\mu_{A}-\mu_{B}\right) / v_{s}\right)$ that is conjugate to the composition variable.

Within the mean-field framework, the equilibrium profiles of the electrostatic potential, ion concentrations, and composition are obtained from extremalization of the grand potential, which results in the following equations:

$$
\begin{gathered}
\varepsilon_{0} \nabla \cdot(\varepsilon \nabla \psi)=-\rho, \\
c_{+}=\lambda_{+} \exp \left(-\beta e z_{+} \psi-\beta u_{+}\right), \\
c_{-}=\lambda_{-} \exp \left(\beta e z_{-} \psi-\beta u_{-}\right), \\
\mu_{s}=\frac{k T}{v_{s}}[\ln \phi-\ln (1-\phi)+\chi(1-2 \phi)] \\
-k T\left[\nabla \cdot(C \nabla \phi)-\frac{1}{2} \frac{\partial C}{\partial \phi}(\nabla \phi)^{2}\right] \\
-\frac{\varepsilon_{0}}{2} \frac{\partial \varepsilon}{\partial \phi}(\nabla \psi)^{2}+c_{+} \frac{\partial u_{+}}{\partial \phi}+c_{-} \frac{\partial u_{-}}{\partial \phi},
\end{gathered}
$$

where $\lambda_{+}$and $\lambda_{+}$are the fugacity of cations and anions, respectively. From Eq. (2.7), we can see a shift of the exchange chemical potential caused by the electrostatic interaction and the ion solvation. Based on Eqs. (2.4)-(2.6), the equilibrium grand potential density can be rearranged to

$$
g(\mathbf{r})=-\frac{\varepsilon_{0} \varepsilon(\phi)}{2}(\nabla \psi)^{2}-k T\left(c_{+}+c_{-}\right)+g_{s},
$$

which can be viewed as consisting of three contributions: the electrostatic interaction, the ion distribution, and the solution part. The interfacial tension for the planar interface is the excess grand potential per unit area,

$$
\gamma=\int d z\left[g(z)-g^{\infty}\right],
$$

where $g^{\infty}$ is the grand potential density in the bulk phase, which is equal for the two coexisting bulk phases, and integration is along the normal of the interface.

Substituting Eqs. (2.5) and (2.6) into Eq. (2.4) results in the following solvation-energy augmented PoissonBoltzmann equation: ${ }^{12}$

$$
\begin{array}{r}
-\varepsilon_{0} \nabla \cdot(\varepsilon \nabla \psi)=e \lambda_{+} z_{+} \exp \left(-\beta e z_{+} \psi-\beta u_{+}\right) \\
-e \lambda_{-} z_{-} \exp \left(\beta e z_{-} \psi-\beta u_{-}\right) .
\end{array}
$$

This equation captures the effect of solvation energy on the electrostatic potential and ion distribution at the mean-field level.

An immediate application of Eq. (2.10) is the determination of the electrostatic potential difference between two coexisting bulk phases (also called Galvani potential). Equation
(2.10) for the bulk (i.e., $\nabla \psi=0$ ) combined with the requirement of charge neutrality gives the Galvani potential $\psi_{D}$ as

$$
\begin{aligned}
\psi_{D} & =\psi_{\beta}-\psi_{\alpha} \\
& =\frac{1}{\left(z_{+}+z_{-}\right) e}\left[\left(u_{+, \alpha}-u_{+, \beta}\right)-\left(u_{-, \alpha}-u_{-, \beta}\right)\right] .
\end{aligned}
$$

From Eq. (2.11), we see that a finite potential difference develops if the cations and anions have unequal solvation energy. The difference in solvation energy also gives rise to an unequal partition of ions between the two coexisting bulk phases,

$$
\begin{aligned}
\frac{c_{+, \alpha}^{\infty}}{c_{+, \beta}^{\infty}} & =\frac{c_{-, \alpha}^{\infty}}{c_{-, \beta}^{\infty}} \\
& =\exp \left[\frac{\beta z_{-}\left(u_{+, \beta}-u_{+, \alpha}\right)+\beta z_{+}\left(u_{-, \beta}-u_{-, \alpha}\right)}{z_{+}+z_{-}}\right] .
\end{aligned}
$$

These results were previously derived by Onuki ${ }^{22,23}$ and Kung et al. ${ }^{31}$

To solve the coupled equations (2.4)-(2.7) for a spatially inhomogeneous system, we need to specify the expression for the solvation energy. In Ref. 12, the solvation energy is shown to consist of a nonuniversal part accounting for the local interaction between the ion and the solvent, and a universal part due to correlations at longer length scales. For the present treatment at the mean-field level, we ignore the long length scale effects and retain only the nonuniversal part of the solvation energy which takes the form of a local Born energy, ${ }^{28}$

$$
u_{+}=\frac{z_{+}^{2} e^{2}}{8 \pi a_{+} \varepsilon_{0} \varepsilon(\phi)} \quad \text { and } \quad u_{-}=\frac{z_{-}^{2} e^{2}}{8 \pi a_{-} \varepsilon_{0} \varepsilon(\phi)},
$$

where $a_{+}$and $a_{-}$are, respectively, the radius of the cations and anions. The local dielectric constant of the solvent mixture is assumed to be a simple volume fraction weighted average of its two components, ${ }^{29}$

$$
\varepsilon(\phi)=\varepsilon_{A}(\phi)+\varepsilon_{B}(1-\phi) .
$$

We note that other mixing rules, such as that based on the Clausius-Mossotti equation, ${ }^{30}$ can also be used. However, qualitatively similar results are expected. ${ }^{33}$ In spite of its simplicity, the Born model captures the essential feature of the solvation energy that an ion dissolved in a more polar medium has lower free energy than in a less polar medium. More importantly, the Born solvation energy can be different for cations and anions. This is the key factor that causes the formation of the Galvani potential and charge separation near an interface.

\section{B. Analytical solution for the case of sharp interface}

Equations (2.4)-(2.6) (or equivalent Eq. (2.10)) and (2.7) are coupled and nonlinear, which require numerical solution in general. However, an idealized case where solvent A and 
B form an infinitely sharp interface permits analytical solution for the electrostatic potential profile and ion concentration profile. We briefly discuss the behavior of the excess interfacial tension due to salt ions for this idealized case because the results help understand and provide a basis for comparison with, results for the case of finite interfacial width to be described later.

The composition as well as the dielectric profiles for the sharp interface model are just step functions. The electrostatic potential and ion profiles in this case were first studied by Nielssen and Verwey, ${ }^{32}$ and more recently by Kung et al. ${ }^{31}$ We refer the readers to these references for the details and merely present the results. We use a system of coordinates where the interface between media coincides with the $\mathrm{z}=0$ plane and the A-rich phase resides in the region $z<0$. We assume $a_{+}<a_{-}$throughout this paper. For monovalent salts, the nondimensionalized electrostatic potential $\bar{\psi}=\beta e \psi$ is given by

$$
\bar{\psi}(z)=-4 \tanh ^{-1}\left[I_{\alpha} \exp \left(\kappa_{\alpha} z\right)\right] \text { for } z<0,
$$

$$
\bar{\psi}(z)=\bar{\psi}_{D}+4 \tanh ^{-1}\left[I_{\beta} \exp \left(-\kappa_{\beta} z\right)\right] \text { for } z>0
$$

where $\kappa_{\alpha}=\sqrt{2 c_{\alpha}^{\infty} / \bar{\varepsilon}_{A}}$ and $\kappa_{\beta}=\sqrt{2 c_{\beta}^{\infty} / \bar{\varepsilon}_{B}}$ are the inverse of the Debye screening length in the two bulk regions, with $c_{\alpha}^{\infty}$ and $c_{\beta}^{\infty}$ the salt concentration in solvent $\mathrm{A}$ and $\mathrm{B}$, respectively, far away from the interface, and $\bar{\varepsilon}=\varepsilon_{0} \varepsilon /\left(\beta e^{2}\right)$ the nondimensionalized dielectric constant. $I_{\alpha}$ and $I_{\beta}$ are integration constants determined from the boundary conditions on $\bar{\psi}$ at the interface; they are given by

$$
\begin{aligned}
I_{\alpha} & =\frac{\sqrt{1+\tau^{2}+2 \tau \cosh \left(\bar{\psi}_{D} / 2\right)}-1-\tau \cosh \left(\bar{\psi}_{D} / 2\right)}{\tau \sinh \left(\bar{\psi}_{D} / 2\right)}, \\
I_{\beta} & =\frac{\sqrt{1+\tau^{2}+2 \tau \cosh \left(\bar{\psi}_{D} / 2\right)}-\tau-\cosh \left(\bar{\psi}_{D} / 2\right)}{\sinh \left(\bar{\psi}_{D} / 2\right)},
\end{aligned}
$$

where $\tau=\sqrt{\varepsilon_{B} c_{\beta}^{\infty} /\left(\varepsilon_{A} c_{\alpha}^{\infty}\right)}$ and is independent of the salt concentration as can be seen from Eq. (2.12). Based on Eqs. (2.5) and (2.6), the concentration profiles for cations and anions can be straightforwardly calculated as

$$
\begin{aligned}
& c_{ \pm}(z)=c_{\alpha}^{\infty}\left(\frac{1 \pm I_{\alpha} \exp \left(\kappa_{\alpha} z\right)}{1 \mp I_{\alpha} \exp \left(\kappa_{\alpha} z\right)}\right)^{2} \text { for } z<0, \\
& c_{ \pm}(z)=c_{\beta}^{\infty}\left(\frac{1 \mp I_{\beta} \exp \left(-\kappa_{\beta} z\right)}{1 \pm I_{\beta} \exp \left(-\kappa_{\beta} z\right)}\right)^{2} \text { for } z>0 .
\end{aligned}
$$

In Eqs. (2.19) and (2.20), we can see that the concentration of cations is different from the concentration of anions at the same position. The difference in concentration becomes more significant when $|z|$ gets smaller (i.e., approaching the interface). The charge separation is caused by the spatial varying electrostatic potential, which in turn originates from the unequal solvation energy between cations and anions. In addi- tion, the concentration of the cations is higher than the anions (i.e., net negative charge accumulates) on the solvent-A side, while lower than the anions (i.e., net positive charge accumulates) on the solvent-B side. Therefore, an electrostatic double layer forms around the sharp interface. As indicated in Eqs. (2.19) and (2.20), the length scale of the electrostatic double layer is characterized by the corresponding Debye screening length on each side: the layer on solvent-B side is thicker than the layer on solvent-A side because $\kappa_{\alpha}^{-1}>\kappa_{\beta}^{-1}$. The thickness of the electrostatic double layer increases as the electrolyte solution becomes dilute.

Equations (2.19) and (2.20) also lead to the following inequality:

$$
\begin{aligned}
& c_{+}(z)+c_{-}(z)>2 c_{\alpha}^{\infty}=c_{+, \alpha}^{\infty}+c_{-, \alpha}^{\infty} \text { for } z<0, \\
& c_{+}(z)+c_{-}(z)>2 c_{\beta}^{\infty}=c_{+, \beta}^{\infty}+c_{-, \beta}^{\infty} \text { for } z>0,
\end{aligned}
$$

which indicates that the total ion concentration around the interface is higher than that in the bulk region; in other words, there is an excess of ions accumulating at the sharp interface. The charge separation, electrostatic double layer, and excess ion adsorption at the interface are shown in Fig. 1.

We note that similar expressions of electrostatic potential and ion concentration were given in the work of Kung et $a l .{ }^{31}$ However, in this section, we emphasize their effect on the excess interfacial tension of the sharp interface and will compare it to the results of the diffuse interface in Sec. IV. The change in the interfacial tension for the sharp interface $\gamma_{s h}$ can be divided into the electrostatic interaction part $\left(\gamma_{1}\right)$ and the ion distribution part $\left(\gamma_{2}\right)$ based on Eqs. (2.8) and (2.9),

$$
\begin{aligned}
\gamma_{s h}= & \gamma_{1}+\gamma_{2} \\
= & -k T \int_{-\infty}^{\infty} d z\left[(\bar{\varepsilon} / 2)(d \bar{\psi} / d z)^{2}\right] \\
& -k T \int_{-\infty}^{\infty} d z\left(c_{+}+c_{-}-c_{+}^{\infty}-c_{-}^{\infty}\right) .
\end{aligned}
$$

From the expressions of the electrostatic potential and the ion concentration, the two contributions to the interfacial tension can be obtained by some straightforward algebra. The results are

$$
\begin{aligned}
\gamma_{1} & =\gamma_{2}=-2 k T \kappa_{\alpha} \bar{\varepsilon}_{A}\left[1+\tau-\sqrt{1+\tau^{2}+2 \tau \cosh \left(\bar{\psi}_{D} / 2\right)}\right] \\
& \sim-\left(c_{\alpha}^{\infty}\right)^{1 / 2},
\end{aligned}
$$

where $\gamma_{1}$ and $\gamma_{2}$ are equal and both are negative. $\gamma_{1}<0$ can be easily seen in Eq. (2.23), whereas $\gamma_{2}<0$ comes from the accumulation of ions at the sharp interface. Therefore, the interfacial tension for a sharp interface decreases as the square root of the salt concentration $\left(\gamma_{s h} \sim-\left(c_{\alpha}^{\infty}\right)^{1 / 2}\right)$ at the meanfield level. This result is in agreement with the theoretical results of Nichols and Pratt ${ }^{20}$ and also with the experimental results measured by Guest and Lewis ${ }^{17}$ at low salt concentrations. However, the results for this idealized composition profile fails to explain the linear increase with salt concentration at higher concentrations. More realistic description of the composition profile (such as Eq. (2.7)) is necessary to 

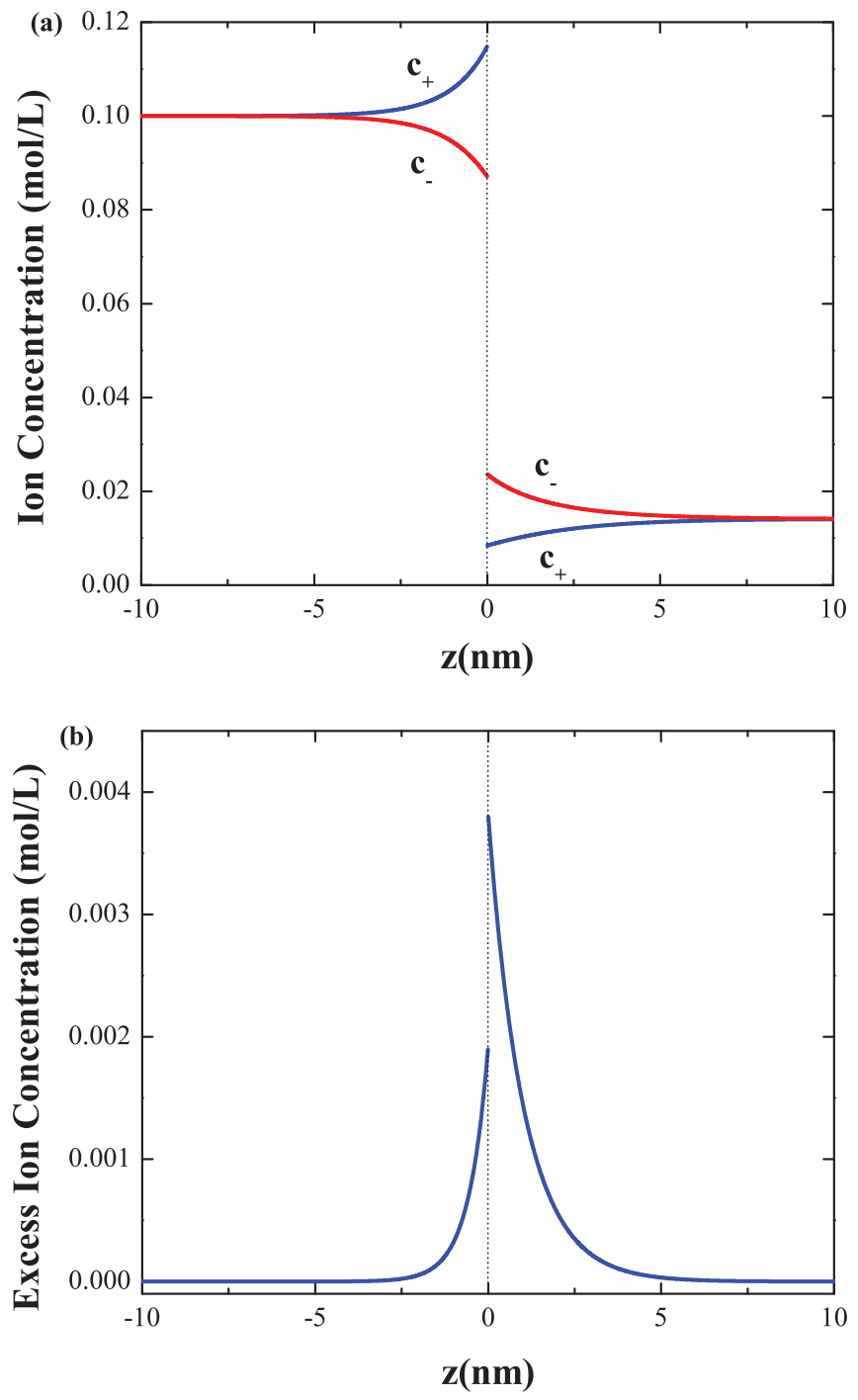

FIG. 1. (a) The distribution of ions ( $c_{+}$and $\left.c_{-}\right)$and (b) excess ion concentration $\left(c_{+}+c_{-}-c_{+}^{\infty}-c_{-}^{\infty}\right)$ between two immiscible solvents with $\varepsilon_{\mathrm{A}}=80$, $\varepsilon_{\mathrm{B}}=40, c_{\alpha}^{\infty}=0.1 \mathrm{~mol} / \mathrm{L}, z_{+}=z_{-}=1, a_{+}=0.1 \mathrm{~nm}$, and $a_{-}=0.2 \mathrm{~nm}$. The dotted line indicates location of the sharp interface.

explain the complex behavior of the interfacial tension in the full range of the salt concentration.

\section{BULK THERMODYNAMICS}

The equilibrium interfacial profile is formed under the condition of equilibrium between two coexisting phases, which will in general be shifted by the addition of salt ions. Therefore, before discussing the interfacial properties, we first examine the thermodynamics of the bulk electrolyte solution in this section. The shift in the spinodal has been studied previously by one of us. ${ }^{33}$ Here we calculate the full phase diagram.

It can be seen from Eq. (2.7) that the electrostatic interaction and the ion solvation alter the chemical potential of the solvent. The expression of the Born model (Eq. (2.13)) indicates that ions have lower solvation energy in the medium with higher dielectric constant, implying a higher solubility. The tendency for ions to be preferentially solvated by the solvent with the higher dielectric constant creates a driving force for phase separation of the two solvent components. On the other hand, the translational entropy of ions favors a uniform distribution, i.e., a single phase state.

To quantitatively analyze this effect, we write the exchange chemical potential and the grand potential density in the bulk phase according to Eqs. (2.7) and (2.8) as

$$
\begin{gathered}
\mu_{s}=\frac{k T}{v_{s}}[\ln \phi-\ln (1-\phi)+\chi(1-2 \phi)] \\
-\left[c_{+}^{\infty}(\phi) u_{+}(\phi)+c_{-}^{\infty}(\phi) u_{-}(\phi)\right] \frac{\Delta \varepsilon}{\varepsilon(\phi)}, \\
g^{\infty}=\frac{k T}{v_{s}}[\phi \ln \phi+(1-\phi) \ln (1-\phi)+\chi \phi(1-\phi)] \\
-k T\left[c_{+}^{\infty}(\phi)+c_{-}^{\infty}(\phi)\right]-\mu_{s} \phi,
\end{gathered}
$$

where the concentration of the cations and anions is calculated from Eqs. (2.5) and (2.6). Theoretically, phase equilibrium of the solvents mixture is best studied at a given chemical potential of cations and anions. The choice of the reservoir is arbitrary; for convenience, we choose the reservoir to be a salt solution in the pure solvent A. The chemical potential of ions is controlled by adjusting the salt concentration in the reservoir.

The phase boundary (binodal line) is determined by the respective equality of the chemical potential and the grand potential density in the two coexisting phases (denoted by $\alpha$ and $\beta$, respectively): $\mu_{s, \alpha}=\mu_{s, \beta}$ and $g_{\alpha}^{\infty}=g_{\beta}^{\infty}$. The spinodal is determined by the vanishing of the second derivative of $g^{\infty}$ with respect to the composition, i.e., $\partial^{2} g^{\infty} / \partial \phi^{2}=0$. The spinodal condition is found to be

$$
\frac{1}{v_{s}}\left(\frac{1}{\phi}+\frac{1}{1-\phi}\right)=2(\chi+\Delta \chi),
$$

where $\Delta \chi$ is the change of the interaction parameter resulting from adding the salt, given by

$$
\begin{aligned}
\Delta \chi= & c^{\infty}(\phi)\left[\beta u_{+}(\phi)+\beta u_{-}(\phi)\right]\left(\frac{\Delta \varepsilon}{\varepsilon(\phi)}\right)^{2} \\
& \times\left[\frac{z_{-} \beta u_{+}(\phi)+z_{+} \beta u_{-}(\phi)}{z_{+}+z_{-}}-2\right] .
\end{aligned}
$$

Here, $\Delta \chi>0$ indicates that the miscibility between the two solvents decreases due to addition of salt, whereas $\Delta \chi<0$ indicates increased miscibility. We see that $\Delta \chi$ is proportional to the salt concentration. We note that the sign of $\Delta \chi$ is determined by the terms in the square brackets in Eq. (3.4), reflecting the competition between the solvation energy and translational entropy.

In most cases, the solvation energy of cations and anions is more than $10 k T$, which means $\Delta \chi$ is positive, resulting in phase separation at lower values of $\chi$. Figure 2(a) shows that both the binodal and the spinodal move downward in the ioncontaining system compared to the ion-free system. When the solvation energy is less than $2 k T$, a negative $\Delta \chi$ is obtained. From the Born model, this condition can be accomplished by high dielectric constant, high temperature, and/or large ion radius. Figure 2(b) shows a phase diagram at high dielectric 

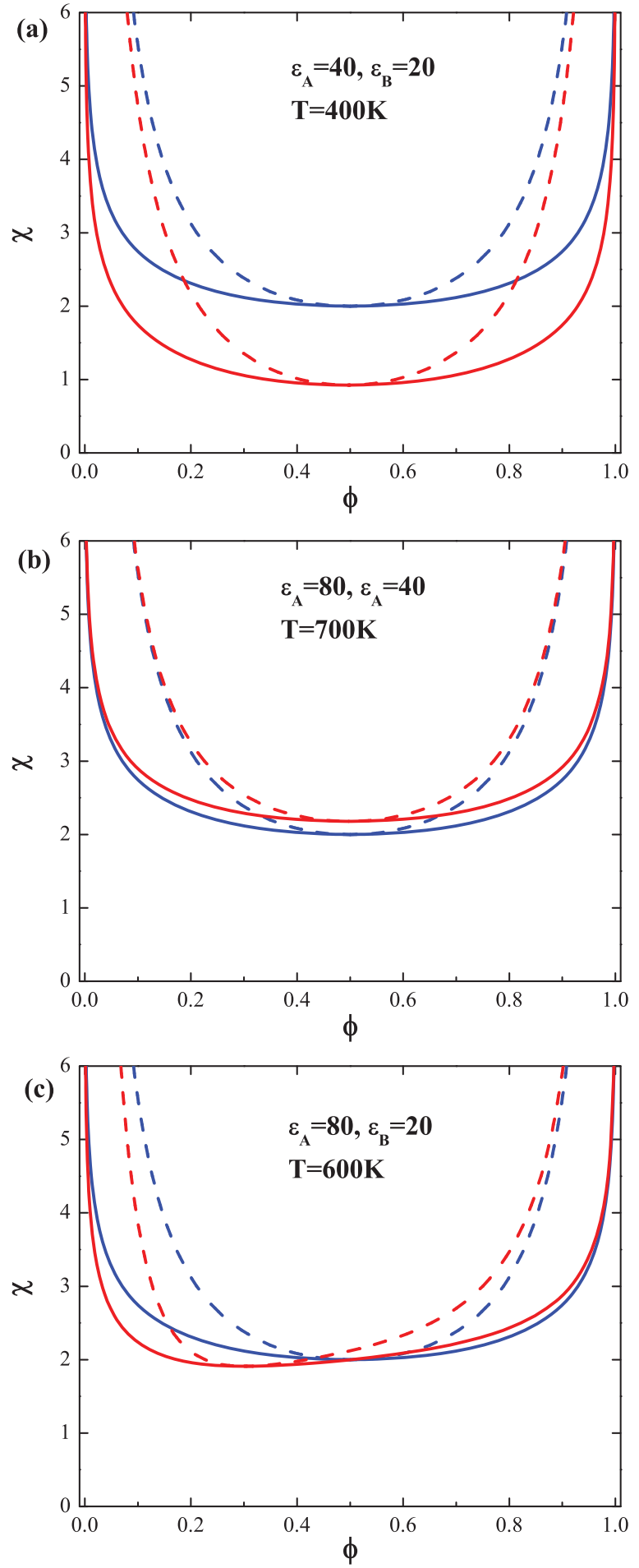

FIG. 2. Effect of the ion solvation on the phase diagram of the binary mixture. Solid lines are the binodal and dashed lines are the spinodal. The red lines are for the ion-containing system and the blue lines are for the ionfree system. The salt concentration in the reservoir is $2 \mathrm{~mol} / \mathrm{L}, z_{+}=z_{-}=1$, $a_{+}=0.1 \mathrm{~nm}, a_{-}=0.3 \mathrm{~nm}$, and $a_{s}=0.5 \mathrm{~nm}$.

constant and high temperature; miscibility is enhanced in this case. Moreover, we can see that the effect of the ion solvation on miscibility is more significant for the case of positive $\Delta \chi$ than negative $\Delta \chi$ because the correction depends not only on the difference in the dielectric constant, but also on the dielec- tric constant itself. Equation (3.4) indicates that the correction becomes less noticeable as $\varepsilon$ increases.

In the case where the dielectric constant of one solvent is high and of the other is low, the effect of adding salt on miscibility depends on the composition of the mixture. Miscibility increases if the high dielectric constant component is the majority, while it decreases if the low dielectric constant component is the majority. This kind of phase diagram is shown in Fig. 2(c).

\section{INTERFACIAL PROPERTIES}

The interfacial profiles of electrostatic potential, ion concentration, and composition are obtained by simultaneously solving Eqs. (2.4)-(2.7) with boundary conditions provided by the bulk thermodynamics discussed above. Compared to the case of sharp interface, the additional Eq. (2.7) gives rise to a finite composition gradient or, physically, a diffuse interface. For the mixture well below the critical temperature, the width of the diffuse interface scales as $C^{-1}$. Thus, $C^{-1}$ becomes another length scale of the system in addition to the Debye screening length $\left(\kappa^{-1}\right)$ that scales the electrostatic double layer. The interplay between these two length scales makes the interfacial structure and properties more complex, which we discuss below.

\section{A. Interfacial structure}

As discussed in Sec. II B, the electrostatic double layer is formed because of the finite potential difference between the two coexistent phases. Charge separation occurs within the electrostatic double layer. Excess ions accumulate near the interface. On the other hand, the composition of the mixture changes within the diffuse interface, which results in a significant change of the solvation energy. Ions will be preferentially distributed in the region of lower solvation energy as discussed in Sec. III. The interfacial structure is determined by these two effects.

From the expression of $C$, we see that the length scale of the diffuse interface is approximately several $a_{s}$, that is on the order of $1 \mathrm{~nm}$. However, the Debye screening length can vary from nanometer to micrometer, depending on salt concentration. Figure 3 shows the structure of the interface for the electrolyte solution with intermediate salt concentration $\left(c_{\alpha}^{\infty}=0.01 \mathrm{~mol} / \mathrm{L}\right)$. From the composition profile in Fig. 3(a), we can see a diffuse interface with the width of $7 \mathrm{~nm}$ between the two coexisting bulk solutions. The change of the electrostatic potential profile is smoother than the composition profile, which indicates that the electrostatic double layer is significantly wider than the composition interface $\left(C^{-1}<\kappa^{-1}\right)$.

The concentration of cations and anions and the total ion concentration $\left(c_{+}+c_{-}\right)$are shown, respectively, in Figs. 3(b) and 3(c), from which we can see the behavior of ion distribution in different regions. Beyond the electrostatic double layer (i.e., $|z|>\kappa^{-1}$ ), the concentration of cations and anions maintain its bulk value. Charge neutrality is satisfied in this bulk region (as denoted by 1 and $1^{\prime}$ in Fig. 3(c)). In the 

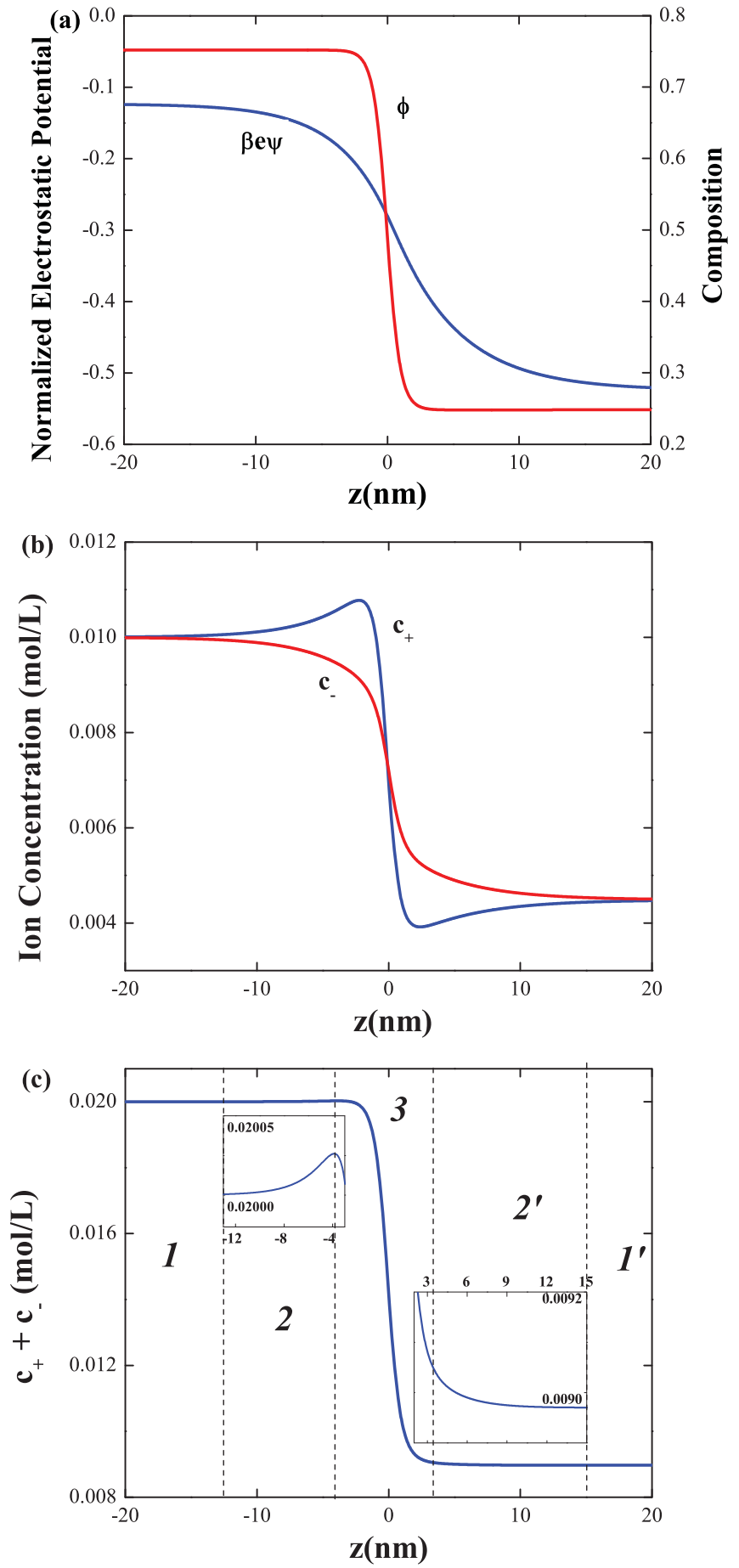

FIG. 3. The interfacial structure for the electrolyte solution with $c_{\alpha}^{\infty}$ $=0.01 \mathrm{~mol} / \mathrm{L}$, (a) profiles of normalized electrostatic potential and composition, (b) the concentration of cations and anions, and (c) total ion distribution. The insets in (c) show the excess ion accumulation in regions 2 and $2^{\prime}$. $\varepsilon_{\mathrm{A}}=80, \varepsilon_{\mathrm{B}}=40, z_{+}=z_{-}=1, a_{+}=0.1 \mathrm{~nm}, a_{-}=0.2 \mathrm{~nm}, a_{s}=0.5 \mathrm{~nm}$, $\chi=2.2$, and $T=400 \mathrm{~K}$.

intermediate region that is inside the electrostatic double layer but outside the diffuse interface $\left(\kappa^{-1}>|z|>C^{-1}\right)$, the composition is nearly constant while the electrostatic potential changes smoothly. The behavior of the ion distribution in this region (denoted by 2 and $2^{\prime}$ as in Fig. 3(c)) is qualitatively the same as the case of the sharp interface. Charge separation becomes more significant as $|z|$ decreases. There are also ex- cess ions accumulating (see the insets of Fig. 3(c)) in this region. Within the diffuse interface $\left(|z|<C^{-1}\right)$, the composition profile changes more dramatically than the electrostatic potential profile. Solvation energy is the dominant effect on the ion distribution in this region (denoted by 3 as in Fig. $3(\mathrm{c}))$. The concentration of both cations and anions decreases when entering from region 2 because of the penetration of the solvent molecules with low dielectric constant in the diffuse interface. Similarly, the ion concentration increases when entering from region $2^{\prime}$. To calculate the net change of the ion concentration in region 3 compared to the bulk region, the exact position of the interface is needed. Onuki ${ }^{22}$ pointed out that the ions are repelled from the diffuse interface by defining the interface position with the Gibbs construction on the composition profile. ${ }^{34}$ With the composition profile, cation and anion profiles all different, an unambiguous Gibbs construction is not obviously possible. Nevertheless, the conclusion that ions are slightly depleted in region 3 still stands, which is reflected by the behavior of the interfacial tension as will be discussed in Sec. IV B.

The length scale of the electrostatic double layer strongly depends on the salt concentration, which leads to differences in the interfacial structure for the concentrated and the dilute electrolyte solution. Figures 4 and 5 show the profiles of electrostatic potential and composition, and the ion distribution for these two cases $\left(c_{\alpha}^{\infty}=1 \mathrm{~mol} / \mathrm{L}\right.$ and $c_{\alpha}^{\infty}=0.001$ $\mathrm{mol} / \mathrm{L}$ ). For $c_{\alpha}^{\infty}=1 \mathrm{~mol} / \mathrm{L}$, both the electrostatic double layer and the diffuse interface have a length scale of a few nanometers $\left(\kappa^{-1} \cong C^{-1}\right)$. Regions 2 and $2^{\prime}$ shrink. The charge separation is greatly inhibited as shown in Fig. 5(a). The change of the solvation energy plays the main role in determining the ion distribution, which leads to ions being repelled from the interfacial area. For the case of very dilute electrolyte solution $\left(c_{\alpha}^{\infty}=0.001 \mathrm{~mol} / \mathrm{L}\right)$, the electrostatic double layer is about 100 times wider than the diffuse interface $\left(\kappa^{-1} \gg C^{-1}\right)$. From

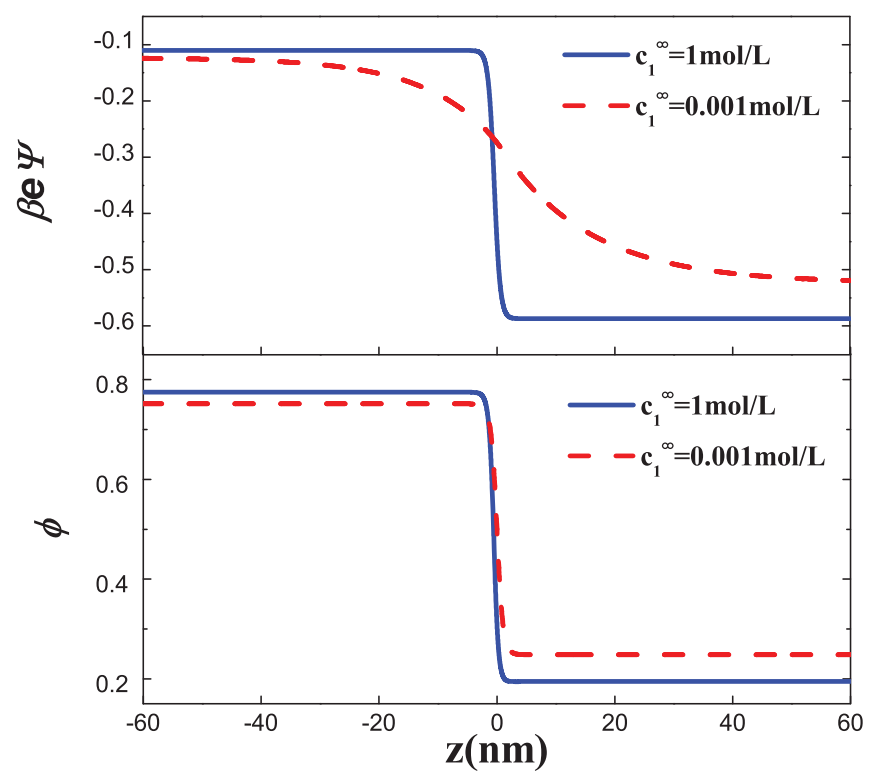

FIG. 4. Profiles of normalized electrostatic potential and composition for $c_{\alpha}^{\infty}=1 \mathrm{~mol} / \mathrm{L}$ and $c_{\alpha}^{\infty}=0.001 \mathrm{~mol} / \mathrm{L} . \varepsilon_{\mathrm{A}}=80, \varepsilon_{\mathrm{B}}=40, z_{+}=z_{-}=1$, $a_{+}=0.1 \mathrm{~nm}, a_{-}=0.2 \mathrm{~nm}, a_{s}=0.5 \mathrm{~nm}, \chi=2.2$, and $T=400 \mathrm{~K}$. 


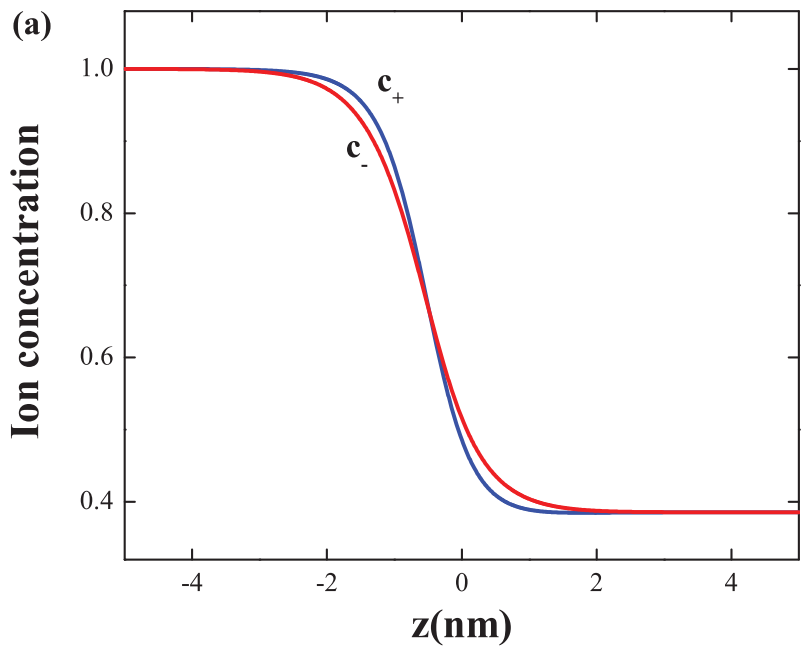

(b)

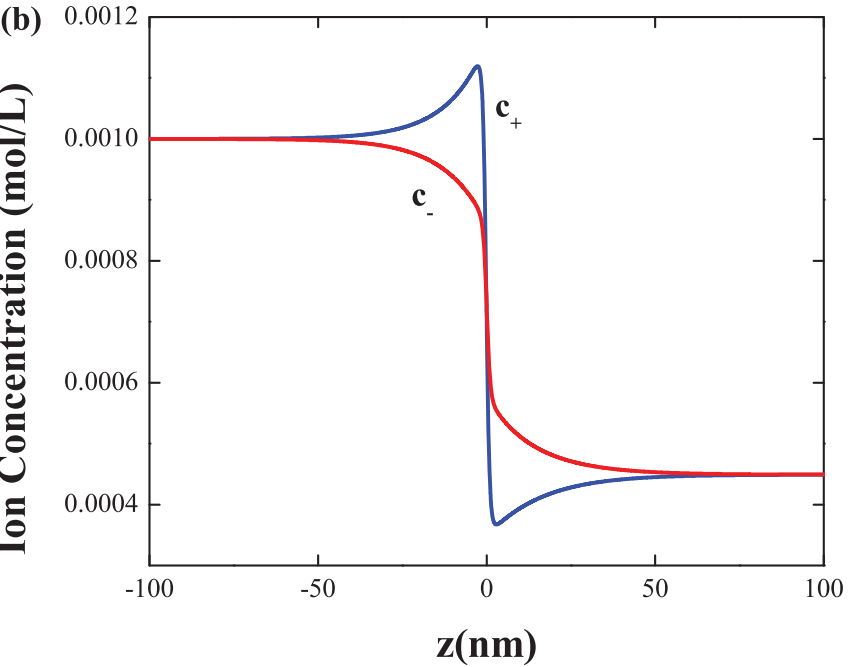

FIG. 5. The concentration profiles of cations and anions for (a) $c_{\alpha}^{\infty}$ $=1 \mathrm{~mol} / \mathrm{L}$ and (b) $c_{\alpha}^{\infty}=0.001 \mathrm{~mol} / \mathrm{L} . \varepsilon_{\mathrm{A}}=80, \varepsilon_{\mathrm{B}}=40, z_{+}=z_{-}=1, a_{+}$ $=0.1 \mathrm{~nm}, a_{-}=0.2 \mathrm{~nm}, a_{s}=0.5 \mathrm{~nm}, \chi=2.2$, and $T=400 \mathrm{~K}$.

the view of the electrostatic potential which changes very smoothly, the composition profile behaves as a step function. The behavior of ion distribution is similar to the case of sharp interface, seen from the similarity between Figs. 5(b) and 1(a). Excess ions accumulate in the interface area.

\section{B. Interfacial tension}

The interfacial structure of the electrolyte solution mixture has direct consequences on the behavior of the interfacial tension. The excess interfacial tension can be divided into two parts,

$$
\Delta \gamma=\gamma-\gamma_{0}=\gamma_{1}+\gamma_{2},
$$

where $\gamma_{0}$ is the interfacial tension of the salt-free system $\left(\gamma_{0}\right.$ $\left.=\int d z\left[g_{0}-g_{0}^{\infty}\right]\right)$. Based on Eq. (2.8), $\gamma_{1}$ and $\gamma_{2}$ are expressed, respectively, as

$$
\gamma_{1}=-k T \int_{-\infty}^{\infty} d z\left[(\bar{\varepsilon} / 2)(d \bar{\psi} / d z)^{2}\right]
$$

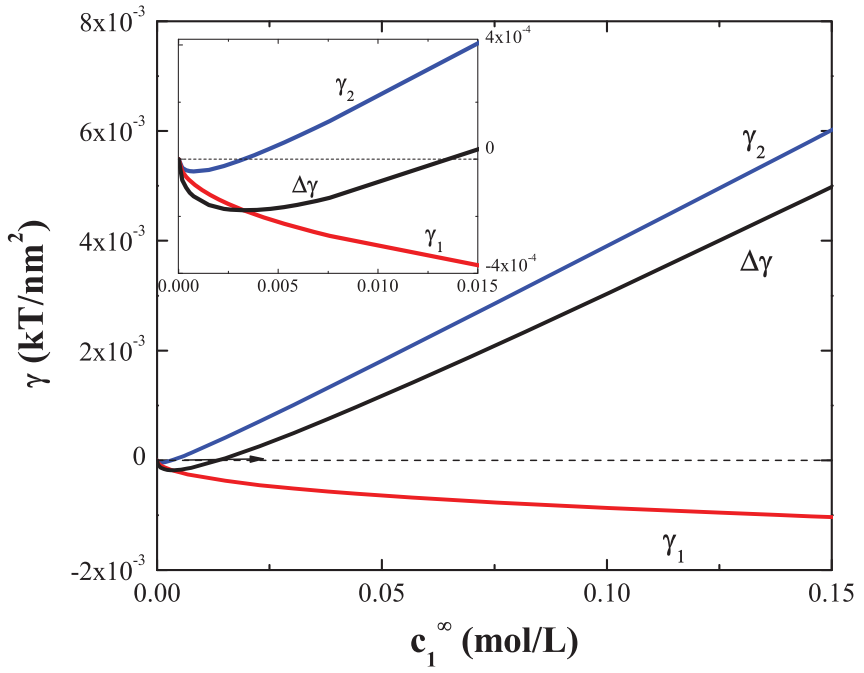

FIG. 6. $\gamma_{1}, \gamma_{2}$, and $\Delta \gamma$ as functions of $c_{\alpha}^{\infty}$. The inset shows the behavior at low concentrations. $\varepsilon_{\mathrm{A}}=80, \varepsilon_{\mathrm{B}}=40, z_{+}=z_{-}=1, a_{+}=0.1 \mathrm{~nm}, a_{-}$ $=0.2 \mathrm{~nm}, a_{s}=0.5 \mathrm{~nm}, \chi=2.2$, and $T=400 \mathrm{~K}$.

$$
\begin{aligned}
\gamma_{2}= & -k T \int_{-\infty}^{\infty} d z\left(c_{+}+c_{-}-c_{+}^{\infty}-c_{-}^{\infty}\right) \\
& +\int_{-\infty}^{\infty} d z\left[\left(g_{s}-g_{s}^{\infty}\right)-\left(g_{0}-g_{0}^{\infty}\right)\right] .
\end{aligned}
$$

Here, $\gamma_{1}$ is due to the electrostatic interactions and $\gamma_{2}$ combines the influences of the ion distribution and the solution free energy. As pointed out by Onuki, ${ }^{22}$ the contribution of the second term in Eq. (4.3) to $\gamma_{2}$ is less important than the first term because the interfacial excess free energy of the ion-containing system is mostly cancelled out by the saltfree system. Therefore, $\gamma_{2}$ is mainly due to the change of ion concentration in the interfacial area compared to the bulk phase $\left(\gamma_{2} \cong-k T \int_{-\infty}^{\infty} d z\left(c_{+}+c_{-}-c_{+}^{\infty}-c_{-}^{\infty}\right)\right)$. From Eqs. (4.2) and (4.3), we see that $\gamma_{1}$ is always negative, while the sign of $\gamma_{2}$ depends on whether there is depletion or enrichment of ions in the interfacial region.

The change of $\gamma_{1}, \gamma_{2}$, and $\Delta \gamma$ as functions of the salt concentration in bulk phase $1\left(c_{\alpha}^{\infty}\right)$ is shown in Fig. 6. Here, $\gamma_{1}$ decreases approximately in proportion to $\left(c_{\alpha}^{\infty}\right)^{1 / 2}$ in the full range of the salt concentration, which is similar to its behavior in the sharp-interface case. $\gamma_{2}$ is positive and increases linearly with $c_{\alpha}^{\infty}$ when the salt concentration is high; this is due to the depletion of ions since the amount of depleted ions is proportional to the bulk salt concentration. This is consistent with the reasoning given in Sec. IV A that, for large $c_{\alpha}^{\infty}$, the repulsion of ions from the diffuse interface dominates over the accumulation of ions in the electrostatic double layer. However, $\gamma_{2}$ becomes negative in the very dilute regime $\left(c_{\alpha}^{\infty}<0.005 \mathrm{~mol} / \mathrm{L}\right)$, shown in the inset of Fig. 6. Excess ions are absorbed into the interfacial region because with the increase in the width of the electrostatic double layer due to decreased salt concentration, the composition profile behaves increasingly like a sharp interface.

With this qualitative understanding of the concentration dependence of $\gamma_{1}$ and $\gamma_{2}$, it is now easy to understand the 


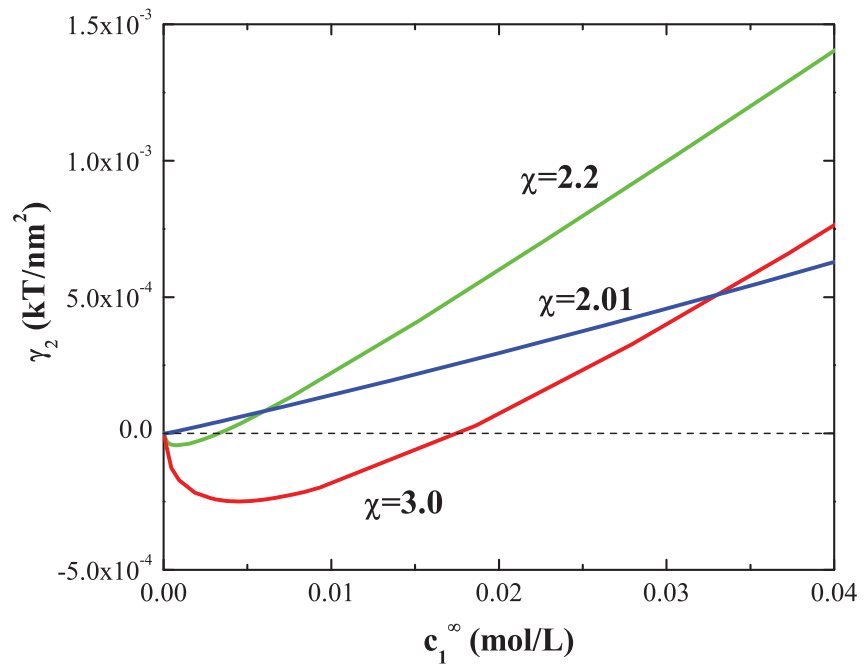

FIG. 7. $\gamma_{2}$ as functions of $c_{\alpha}^{\infty}$ for three different values of $\chi \cdot \varepsilon_{\mathrm{A}}=80, \varepsilon_{\mathrm{B}}$ $=40, z_{+}=z_{-}=1, a_{+}=0.1 \mathrm{~nm}, a_{-}=0.2 \mathrm{~nm}, a_{s}=0.5, \mathrm{~nm}$, and $T$ $=400 \mathrm{~K}$.

behavior of $\Delta \gamma$. At high salt concentration, $\left|\gamma_{1}\right|<\left|\gamma_{2}\right| ; \Delta \gamma$ $\cong \gamma_{2}$, which is positive and increases linearly with the salt concentration. On the other hand, $\left|\gamma_{1}\right|>\left|\gamma_{2}\right|$ at low salt concentration; $\Delta \gamma \cong \gamma_{1}$, which is negative and decreases in proportional to the square root of the salt concentration. The different scaling behaviors of the interfacial tension with respect to the salt concentration (i.e., $\Delta \gamma \sim c_{\alpha}^{\infty}$ at large $c_{\alpha}^{\infty}$; $\Delta \gamma \sim-\left(c_{\alpha}^{\infty}\right)^{1 / 2}$ at small $\left.c_{\alpha}^{\infty}\right)$ is in agreement with the experiment results of the liquid-gas surface tension measured by Petersen and Saykally, ${ }^{14}$ and the liquid-liquid interfacial tension measured by Guest and Lewis. ${ }^{17}$ Moreover, the $\Delta \gamma$ curve has a small negative minimum $\left(\Delta \gamma_{\min } \sim-10^{-3} \gamma_{0}\right)$ in the dilute regime $\left(c_{\alpha}^{\infty} \sim 10^{-3} \mathrm{~mol} / \mathrm{L}\right)$. Both the location of the minimum and its magnitude are in the range of the Jones-Ray effect observed in the experiments.

To further demonstrate the effect of the interfacial structure on the interfacial tension, we investigate the behavior of $\gamma_{2}$ at three different values of the interaction parameter $\chi$, as shown in Fig. 7. The value of $\chi$ determines the degree of phase separation and hence the width of the diffuse interface in combination with $C^{-1}$. As $\chi$ increases from 2.01 to 3 , the width of the diffuse interface decreases from $20 \mathrm{~nm}$ to $2 \mathrm{~nm}$. A narrower interface is less capable of depleting ions. For the case of $\chi=3$, starting at high salt concentration and going in the direction of decreasing concentration, the effect of ion accumulation in the electrostatic double layer increases relative to the effect of ion depletion in the diffuse interface. $\gamma_{2}$ becomes negative at $c_{\alpha}^{\infty}=0.018 \mathrm{~mol} / \mathrm{L}$ as shown in Fig. 7. For small $\chi$, especially near the critical point, the composition interface is quite wide. A larger width for the electrostatic double layer is required to counteract this effect. Thus, $\gamma_{2}$ remains positive even for very dilute electrolyte solution $\left(c_{\alpha}^{\infty} \sim 10^{-5}\right.$ $\mathrm{mol} / \mathrm{L}$ ), as shown for the case of $\chi=2.01 \mathrm{in} \mathrm{Fig.} 7$.

Experimentally, the excess water-air surface tension is known to show a marked specific ion effect that decreases according to the Hofmeister sequence: ${ }^{15,16} \mathrm{~F}^{-}>\mathrm{Cl}^{-}>\mathrm{Br}^{-}$ $>\mathrm{I}^{-}$. Similar dependence of the liquid-liquid interfacial tension on the cations and anions themselves has also been ob-

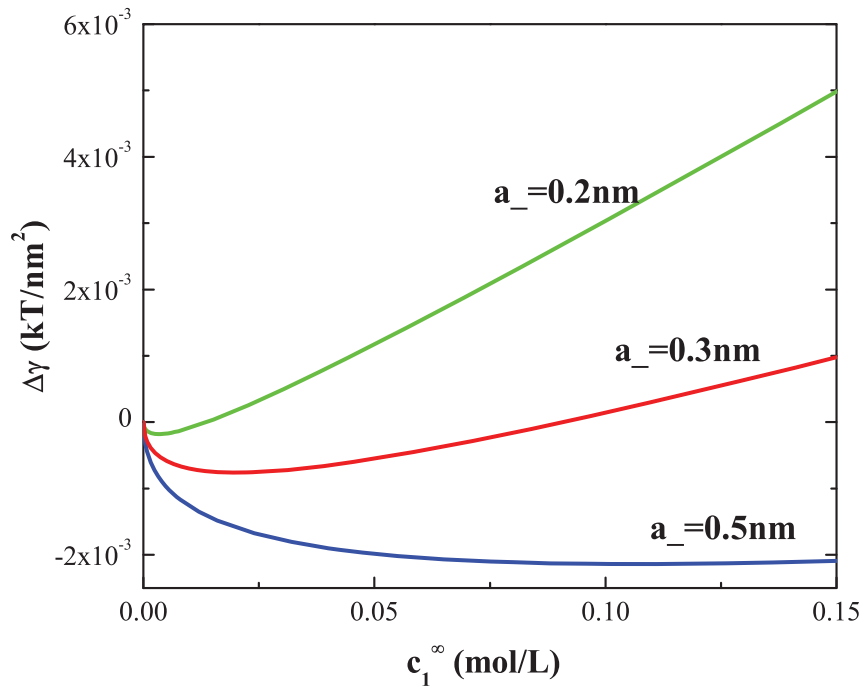

FIG. 8. $\Delta \gamma$ as functions of $c_{\alpha}^{\infty}$ for three different values of $a_{-} . \varepsilon_{\mathrm{A}}=80$, $\varepsilon_{\mathrm{B}}=40 . \quad z_{+}=z_{-}=1, a_{+}=0.1 \mathrm{~nm}, a_{s}=0.5 \mathrm{~nm}, \quad \chi=2.2$, and $T$ $=400 \mathrm{~K}$.

served. Guest and Lewis found that, ${ }^{17}$ for $\mathrm{K}^{+}$as the cation, the interfacial tension between water and dekalin significantly decreases as the anion changes in the sequence of $\mathrm{Cl}^{-}, \mathrm{I}^{-}$, and $\mathrm{SCN}^{-}$. For the latter two anions, $\Delta \gamma$ is even negative up to a quite high salt concentration. This effect can also be explained by our model. The difference between the solvation energy of a specific ion in the two solvents decreases inversely with the ion radius. Let us first change the radius of anions by fixing the radius of cations under the precondition that $a_{+}<a_{-}$. From Eqs. (2.11) and (2.12), we can see that $\left|\psi_{D}\right|$ increases and $c_{\alpha}^{\infty} / c_{\beta}^{\infty}$ decreases as $a_{-}$increases. The increase of $\left|\psi_{D}\right|$ gives rise to a larger gradient of electrostatic potential, which causes $\gamma_{1}$ to become more negative. The decrease of $c_{\alpha}^{\infty} / c_{\beta}^{\infty}$ reduces the amount of ions that are depleted from the diffuse interface, and thus decreases $\gamma_{2} \cdot \gamma_{1}$, and $\gamma_{2}$ change in the same direction, which makes $\Delta \gamma$ decrease as the radius of the anions increases. Figure 8 shows the dependence of the interfacial tension on the radius of anions. For small ions, $\Delta \gamma$ has a linear relationship with the salt concentration in the concentrated region, while for large ions, $\Delta \gamma$ maintains the $-\left(c_{\alpha}^{\infty}\right)^{1 / 2}$ scaling in a wide range of the salt concentration. Similar analysis can also be made for the size change of cations under the condition that $a_{+}<a_{-}$. However, $\gamma_{1}$ and $\gamma_{2}$ change in opposite directions in this case, which weakens the effect of $a_{+}$on $\Delta \gamma$. This is consistent with the general experimental finding that the interfacial tension usually depends more strongly on anions than cations. ${ }^{15}$

We note also that our results are consistent with the findings of Bhatt et al. ${ }^{35}$ who studied the specific-ion effect on the surface tension of aqueous electrolytic solutions using molecular dynamics simulation. In their work, it is found that when water molecules are treated explicitly and the ions are treated simply as charged Lennard-Jones particles, the surface tension shows a larger increase for the $\mathrm{NaF}$ solution than for the $\mathrm{NaCl}$ solution and the simulation data are in good agreement with experiment; this is consistent with the expectation from our theory based on the size dependence in the solvation energy of the ions. On the other hand, the opposite trend is 
obtained when water molecules are treated as a simple dielectric within the primitive model, which does not account for the solvation of ions. We, therefore, conclude that the sizedependent solvation energy of the ions is the key factor in determining the interfacial tension.

\section{SUMMARY AND CONCLUSIONS}

In this paper, we have presented a unified model in the mean-field framework to describe the bulk thermodynamics and interfacial properties of the electrolyte solution mixture, by taking the solvation energy of ions into account. The electrostatic potential, ion concentration, and solvent composition are treated explicitly in our model. At the mean-field level, the solvation energy retains the form of Born energy which is spatially dependent in an inhomogeneous dielectric medium. The Born energy captures a key characteristic of a specific ion by its radius and valency. The difference in the solvation energy between the cations and anions leads to the local charge separation, a finite electrostatic potential difference between two coexisting phases, and the formation of an electrostatic double layer at the interface.

Ion solvation affects the phase equilibrium of the solvent mixture. The miscibility of the mixture can either increase or decrease depending on the relative value between the average solvation energy $\left(z_{-} u_{+}+z_{+} u_{-}\right) /\left(z_{+}+z_{-}\right)$and $2 k T$. When the dielectric constant is low for both solvents, the addition of salt ions decreases the miscibility, while when the dielectric constant for the two solvents is high and at high temperature, the addition of salt ions makes the mixture more miscible. When the dielectric constant of one solvent is high and the other is low, adding salt ions enhances the miscibility if the high-dielectric-constant solvent is the majority component and decreases the miscibility if the low-dielectricconstant solvent is the majority component.

Ion solvation has significant effect on the interfacial structure and properties of the electrolyte solution mixture. The ion distribution in the interfacial area is determined by the competition between the effects of the electrostatic double layer and the diffusive composition interface. The former effect results in accumulation of ions in the interfacial region and is more important in the dilute electrolyte solution, while the latter effect causes ions to be depleted from the interfacial region and dominates at high salt concentration. The interfacial tension, which is a sum of the electrostatic interaction part and the ion excess part, has different scaling behavior as the salt concentration changes. The ion excess is negative and is the dominant contribution in the concentrated regime, leading to an increase in the interfacial tension that scales as $\Delta \gamma \sim c$. On the other hand, the electrostatic interaction part is dominant in the dilute solution, which scales as $\Delta \gamma \sim-c^{1 / 2}$. The interfacial tension has a negative minimum near $1 \mathrm{mM}$, in agreement with the Jones-Ray effect observed in experiments. Moreover, for a fixed cation type, the interfacial tension decreases as the size of anion increases. The specific ion effect can be qualitatively explained by our model when the solvation energy is accounted for.

Our model uses a simple Born form for the solvation energy, with a local dielectric constant given by some composi- tion weighted average of the two liquid components, which is derived within the framework of continuum, linear dielectrics. Such a framework cannot predict the dielectric constant of a mixture in terms of the components, nor does it account for effects, such as dielectric saturation. A more molecularly based approach accounting for the polarizability and permanent dipoles of the solvating molecules would be required to yield a more accurate expression for the solvation energy. ${ }^{36,37}$

In this work, the electrolyte solution is investigated at the mean-field level, where the solvation energy retains the form of Born energy. However, the solvation energy is closely related to the fluctuation in a charged system, which includes both the local Born solvation effect and other long-range effects. A well-known example is the depletion of ions from the water-air interface caused by the image repulsion. To fully account for the solvation energy, Green's function describing the charge correlation needs to be solved for in addition to the modified PB equation, as presented in Ref. 12. Onuki ${ }^{22}$ pointed out that the image interaction can dominate over the local solvation energy if the dielectric inhomogeneity of the two media becomes strong, especially for the liquid-gas and liquid-solid interfaces. The Green's function approach has been used for the sharp-interface model, in both the planar ${ }^{38}$ and curved geometry. ${ }^{39}$ Full fluctuation effects need to be incorporated in future work in order to study the interfacial behavior in these systems.

\section{ACKNOWLEDGMENTS}

This work is in part supported by the National Science Foundation through MRSEC-CALTECH.

${ }^{1} \mathrm{~J}$. Israelachvili, Intermolecular and Surface Forces, 2nd ed. (Academic, London, 1992).

${ }^{2}$ D. Andelman, in Soft Condensed Matter Physics in Molecular and Cell Biology, edited by W. C. K. Poon and D. Andelman, (Taylor and Francis, Boca Raton, Florida, 2000), pp. 97-118.

${ }^{3}$ Y. Levin, Rep. Prog. Phys. 65, 1577 (2002).

${ }^{4}$ B. Alberts, D. Bray, J. Lewis, M. Raff, K. Roberts, and J. D. Watson, Molecular Biology of the Cell, 3rd ed. (Garland, New York, 1994).

${ }^{5}$ I. Borukhov, D. Andelman, and H. Orland, Phys. Rev. Lett. 79, 435 (1997).

${ }^{6}$ R. R. Netz and H. Orland, Euro. Phys. J. E 1, 67 (2000).

${ }^{7}$ Y. Levin and J. E. Flores-Mena, Euro. Phys. Lett. 56, 187 (2001).

${ }^{8}$ M. Z. Bazant, B. D. Storey, and A. A. Kornyshev, Phys. Rev. Lett. 106, 046102 (2011).

${ }^{9}$ M. P. Tosi, in Condensed Matter Physics Aspects of Electrochemistry (World Scientific, Singapore, 1991), p. 68.

${ }^{10}$ M. Rovere and M. P. Tosi, Rep. Prog. Phys. 49, 1001 (1986).

${ }^{11}$ W. M. Gelbart, R. F. Bruinsma, P. A. Pincus, and V. A. Parsegian, Phys. Today 53, 38 (2000).

${ }^{12}$ Z.-G. Wang, Phys. Rev. E 81, 021501 (2010).

${ }^{13}$ G. Jones and W. A. Ray, J. Am. Chem. Soc. 57, 957 (1935); 59, 187 (1937); 63, 288 (1941); 63, 3262 (1941); 64, 2744 (1942).

${ }^{14}$ P. B. Petersen and R. J. Saykally, J. Am. Chem. Soc. 127, 15446 (2005).

${ }^{15}$ M. Boström, W. Kunz, and B. W. Ninham, Langmuir 21, 2619 (2005).

${ }^{16}$ M. Boström, D. R. M. Williams, and B. W. Ninham, Biophys. J. 85, 686 (2003).

${ }^{17}$ W. L. Guest and W. C. M. Lewis, Proc. R. Soc. A 170, 501 (1939).

${ }^{18}$ C. Wagner, Phys. Z. 25, 474 (1924).

${ }^{19}$ L. Onsager and N. N. T. Samaras, J. Chem. Phys. 2, 528 (1933).

${ }^{20}$ A. L. Nichols III and L. R. Pratt, J. Chem. Phys. 80, 6225 (1984).

${ }^{21}$ M. Bier, J. Zwanikken, and R. van Roij, Phys. Rev. Lett. 101, 046104 (2008). 
${ }^{22}$ A. Onuki, Phys. Rev. E 73, 021506 (2006).

${ }^{23}$ A. Onuki, J. Chem. Phys. 128, 224704 (2008).

${ }^{24}$ Y. Levin, Phys. Rev. Lett. 102, 147803 (2009).

${ }^{25} \mathrm{H}$. T. Davis, Statistical Mechanics of Phases, Interfaces, and Thin Films (VCH, New York, 1996).

${ }^{26}$ S. A. Safran, Statistical Thermodynamics of Surfaces, Interfaces, and Membranes (Westview Press, Boulder, CO, 2003).

${ }^{27}$ P. G. de Gennes, J. Chem. Phys. 72, 4756 (1980).

${ }^{28}$ M. Born, Z. Phys. 1, 45 (1920).

${ }^{29}$ Y. Marcus, Ion SolVation (Wiley, New York, 1985).

${ }^{30}$ A. R. Blythe and D. Bloor, Electric Properties of Polymers (Cambridge University Press, Cambridge, New York, 2005).
${ }^{31}$ W. Kung, F. J. Solis, and M. O. de la Cruz, J. Chem. Phys. 130, 044502 (2009).

${ }^{32}$ E. J. W. Verwey and K. F. Niessen, Phil. Mag. 28, 435 (1939).

${ }^{33}$ Z.-G. Wang, J. Phys. Chem. B 112(50), 16205 (2008).

${ }^{34} \mathrm{D}$. Chandler, Introduction to Modern Statistical Mechanics (Oxford University Press, Oxford, 1987).

${ }^{35}$ D. Bhatt, J. Newman, and C. J. Radke, J. Phys. Chem. B 108, 9077 (2004).

${ }^{36}$ A. K. Jha and K. F. Freed, J. Chem. Phys. 128, 034501 (2008).

${ }^{37}$ P. Koehl, H. Orland, and M. Delarue, J. Phys. Chem. B 113, 5694 (2009).

${ }^{38}$ D. S. Dean and R. R. Horgan, Phys. Rev. E 69, 061603 (2004).

${ }^{39}$ R. A. Curtis and L. Lue, J. Chem. Phys. 123, 174702 (2005). 